\title{
Synthesis and Characterization of Polycarbonate Copolymers Containing Benzoyl Groups on the Side Chain for Scratch Resistance
}

\author{
Hohyoun Jang, Jaeseong Ha, Jiho Yoo, Jaeseung Pyo, Kunyoung Choi, \\ Chaekyun Lee, Taewook Ryu, Sungkwun Lee, and Whangi Kim
}

Department of Applied Chemistry, Konkuk University, 322 Danwol, Chungju 380-701, Republic of Korea

Correspondence should be addressed to Whangi Kim; wgkim@kku.ac.kr

Received 18 March 2016; Revised 11 July 2016; Accepted 23 July 2016

Academic Editor: Marta Fernández-García

Copyright (C) 2016 Hohyoun Jang et al. This is an open access article distributed under the Creative Commons Attribution License, which permits unrestricted use, distribution, and reproduction in any medium, provided the original work is properly cited.

\begin{abstract}
The purpose of this study was to enhance the scratch resistance of polycarbonate copolymer by using $3,3^{\prime}$-dibenzoyl-4, $4^{\prime}$ dihydroxybiphenyl (DBHP) monomer, containing benzoyl moieties on the ortho positions. DBHP monomer was synthesized from $4,4^{\prime}$-dihydroxybiphenyl and benzoyl chloride, followed by the Friedel-Craft rearrangement reaction with $\mathrm{AlCl}_{3}$. The polymerizations were conducted following the low-temperature procedure, which is carried out in methylene chloride by using triphosgene, triethylamine, bisphenol-A, and DBHP. The chemical structures of the polycarbonate copolymers were confirmed by ${ }^{1} \mathrm{H}$-NMR. The thermal properties of copolymers were investigated by thermogravimetric analysis and differential scanning calorimetry, and also surface morphologies were assessed by atomic force microscopy. The scratch resistance of homopolymer film $(100 \mu \mathrm{m})$ changed from $6 \mathrm{~B}$ to $1 \mathrm{~B}$, and the contact angle of a sessile water drop onto the homopolymer film also increased.
\end{abstract}

\section{Introduction}

Over the past few decades, the demand for polycarbonate as a major engineering plastic has increased because they have attracted a significant attention for a range of applications in several industrial fields. Polycarbonate is an amorphous, clear polymer that exhibits three key characteristic properties: toughness, transparency, self-extinguishing characteristic, and heat resistance [1-5]. These desirable properties, combined with superior dimensional stability and good electrical resistance, have led to wide applications in automobile parts, electrical parts, optical materials, and steam sterilizable medical equipment. In contrast, to improve the polycarbonate properties such as abrasion and scratch resistance, transparency, nonflammability, and the impact resistance, many endeavors have been reported to overcome these concerns [6-8]. Among them, the vulnerability of polycarbonate is because of antiscratch surface property. Several methods have been investigated to enhance the antiscratch resistance. They generally involve modifications by physical or chemical methods. The surface treatment or blend only modifies a very shallow surface of polymer film and sheet and thus does not change the natural characteristics of polymer. In contrast, the chemical modifications provide the means of permanently altering polymer film and sheet [9-11].

Several methods have been investigated to enhance antiscratch property. (1) The antiscratch property of polycarbonate by grafting and block copolymers with PMMA were transparent in contrast to PC/PMMA blend polymers and had a higher surface hardness [12]. (2) The blends/composites of polycarbonate with PMMA, siloxane, and polysiloxane were developed for increasing the stabilization effect such as photodegradation and toughness. However, these blends/composites show phase-separated morphology with poor interfacial adhesion and poor properties [13-15]. (3) Many researchers studied transparent ultrahydrophobic silica films modified alkoxysilane by the sol-gel process under UV irradiation [16]. Several authors reported to enhance the scratch resistance of polycarbonate with $\mathrm{SiO}_{2}$ and $\mathrm{TiO}_{2}$ using microwave-assisted sol-gel heating $[17,18]$. The morphology of the fabric with alkali treatment and polycarbonate coating was studied using the scanning electron microscopy and 
polarized optical microscopic techniques [19]. (4) The chemical modification such as Parmax containing side carbonyl affects the surface properties including the surface morphology and other properties [20]. Research on the effective modification of polycarbonate is necessary, because the main attraction these days is the thin film application of mobile window replacing tempered glass.

The purpose of this study was to improve the scratch resistance of polycarbonate while retaining its beneficial properties. Thin film polycarbonates are difficult to coat by UV curing, because they are very soft with only $6 \mathrm{~B}$ hardness. In this study, we introduced a new copolymer for enhancing the scratch resistance. The copolymer was designed by bisphenolA and 3,3-dibenzoyl-4,4-dihydroxybiphenyl, and the effect of side functional group was confirmed. The chemical analysis and thermal properties of this polycarbonate containing benzoyl groups on side chain and the waterproof and mechanical properties of the polymers were investigated. The surface morphologies were investigated by waterproof and AFM studies. The experimental data show that all the polymers are quite thermally stable and showed good waterproof and improved scratch resistance.

\section{Experimental}

2.1. Materials. Bisphenol-A, 4,4-biphenol, benzoyl chloride, aluminum chloride, triethylamine (TEA), and triphosgene were purchased from Sigma-Aldrich, Junsei, and Acros chemical companies and used as received. Common reagents such as 1,2-dichlorobenzene, ethyl acetate, acetone, methylene chloride, hexane, and sodium hydroxide were also used as received. TEA was used as a $15 \%(\mathrm{w} / \mathrm{v})$ aqueous solution for synthesizing linear polycarbonate.

2.2. Measurement. NMR spectra were recorded using a Bruker DRX $(400 \mathrm{MHz})$ spectrometer using $\mathrm{CDCl}_{3}$ as the solvent and tetramethylsilane as the internal standard. The intrinsic viscosity $\left(\eta_{\text {inh }}\right)$ was determined at a $0.5 \mathrm{~g} / \mathrm{dL}$ concentration of polycarbonate in methylene chloride with a Cannon-Fenske routine viscometer at $30^{\circ} \mathrm{C}$. Differential scanning calorimetry (DSC) analysis was performed using a Perkin-Elmer DSC 6 at a heating rate of $20^{\circ} \mathrm{C} / \mathrm{min}$ under nitrogen. $T_{g}$ was taken as the midpoint of the inflection observed on the curve of heat capacity versus temperature. Thermogravimetric analysis (TGA) was performed using a Scinco TGA-N 1000 analyzer. The surface morphologies of polycarbonates were observed by atomic force microscopy (AFM). Tapping mode atomic force microscopic observations were performed using a Digital Instrument, Nanoscope (R) IIIA, using microfabricated cantilevers and amplitude set point $0.7785 \mathrm{~V}$. The contact angle was measured by a contact angle analyzer (Phoenix-300, Surface Electro Optics). The volume of the water drop was controlled to $\sim 0.2 \mu \mathrm{L}$ by a microsyringe. The average value of both sides of each drop was considered as the contact angle. Antiscratch was measured by KIPAE Promate ${ }^{\mathrm{TM}} 5000 \mathrm{M}$, and test angle was $45^{\circ}$ with a test load of $1 \mathrm{Kg}$. The transparency was measured by VMS-1 parallel beam-path of SCINCO in the wavelength range $400-1000 \mathrm{~nm}$ to use a tungsten halogen lamp.
2.3. Synthesis of 3,3-Dibenzoyl-4,4'-dihydroxybiphenyl (DBHP). The monomer was prepared by Friedel-Craft reaction. In a $250 \mathrm{~mL}$ round flask, $4,4^{\prime}$-biphenol $(5.0 \mathrm{~g}$, $26.8 \mathrm{mmol}$ ) and aluminum chloride $(17.9 \mathrm{~g}, 134.2 \mathrm{mmol})$ were dissolved in 1,2-dichlorobenzene. To this mixture, benzoyl chloride $(9.3 \mathrm{~mL}, 80.5 \mathrm{mmol}$ ) was slowly added at room temperature and stirred for $18 \mathrm{~h}$ at $160^{\circ} \mathrm{C}$. After the reaction, the liquid was poured in distilled water containing a small amount of hydrochloric acid and methylene chloride was added. The separated organic layer was washed twice with water and evaporated. The solid was recrystallized using ethyl acetate and dried in a vacuum oven at $60^{\circ} \mathrm{C}, \mathrm{MP}: 186^{\circ} \mathrm{C},{ }^{1} \mathrm{H}$ NMR ( $\left.\mathrm{CDCl}_{3}, \mathrm{ppm}, \delta\right): 7.10-7.12$ (s, 2H, o-ArH), 11.94-12.05 (s, 2H, ArOH), 7.72-7.81 (m, 6H, -CO-o-ArH), 7.51-7.67 (m, $8 \mathrm{H}, m, p-\mathrm{ArH})$.

2.4. Synthesis of Linear Polycarbonate (BPA-PC). The polymerization was carried out by the following general procedure at room temperature. Bisphenol $\mathrm{A}(3 \mathrm{~g}, 13 \mathrm{mmol})$ and an aqueous $\mathrm{NaOH}$ solution $(3.25 \mathrm{~g}, 81.2 \mathrm{mmol})$ were added to a $250 \mathrm{~mL}$ three-necked round bottom flask. Triphosgene $(2.67 \mathrm{~g}, 9 \mathrm{mmol})$ dissolved in methylene chloride and TEA $(0.06 \mathrm{~mL})$ were added to the solution and stirred for $2 \mathrm{~h}$ at room temperature. The methylene chloride layer was separated and the polymer solution was washed with distilled water, neutralized with $\mathrm{HCl}$, and precipitated in a mixture of acetone and distilled water before drying in a vacuum oven at $80^{\circ} \mathrm{C}(50: 50 \mathrm{v} / \mathrm{v}), T_{g}=151^{\circ} \mathrm{C},{ }^{1} \mathrm{H} \mathrm{NMR}\left(\mathrm{CDCl}_{3}, \mathrm{ppm}, \delta\right): 1.68$ (s, 6H - $\left.\mathrm{C}\left(\mathrm{CH}_{3}\right)_{2}-\right), 7.15-7.26$ (m, 8H, ArH).

2.5. Synthesis of Polycarbonate Copolymers with Side Benzoyl Group (DBHP-PC). Polycarbonate containing $50 \mathrm{~mol} \%$ of the DBHP moiety was prepared by the low-temperature esterification reaction. Bisphenol-A (3.0 g, $13.1 \mathrm{mmol})$, DBHP $(5.2 \mathrm{~g}, 13.1 \mathrm{mmol})$, and TEA $(10.6 \mathrm{~mL}, 78.8 \mathrm{mmol})$ were dissolved in methylene chloride in a $250 \mathrm{~mL}$ three-necked round bottom flask. Triphosgene $(5.2 \mathrm{~g}, 17.4 \mathrm{mmol})$ dissolved in methylene chloride was dropped slowly in the solution at $0^{\circ} \mathrm{C}$ and stirred for $4 \mathrm{~h}$ at room temperature. The polymer solution was washed several times with distilled water and precipitated in a mixture of acetone and distilled water $(50: 50 \mathrm{v} / \mathrm{v})$ before drying in a vacuum oven at $80^{\circ} \mathrm{C},{ }^{1} \mathrm{H} \mathrm{NMR}\left(\mathrm{CDCl}_{3}, \mathrm{ppm}, \delta\right)$ : 6.97-7.17 (s, 2H, o-ArH), 7.81-7.88 (m, 6H, -CO-o-ArH), 7.517.67 (m, 8H, m, p-ArH), 1.68 (s, 6H - $\left.\mathrm{C}\left(\mathrm{CH}_{3}\right)_{2}-\right), 7.15-7.26(\mathrm{~m}$, $8 \mathrm{H}, \mathrm{ArH})$.

\section{Results and Discussion}

The monomer of DBHP was prepared by the modified literature procedure [17-19]. In the literature, mono- and dibenzoyl biphenols were obtained as the products, and they could be only separated by column chromatography, resulting in a low yield. Therefore, the experimental procedure was exclusively modified to obtain single material dibenzoyl biphenol product, followed by the Friedel-Craft reaction with aluminum chloride and benzoyl chloride. Moreover, the desired monoand dibenzoyl biphenols were recrystallized from ethyl acetate. The esterification of polycarbonate was performed by both the interfacial and low-temperature polymerizations. 


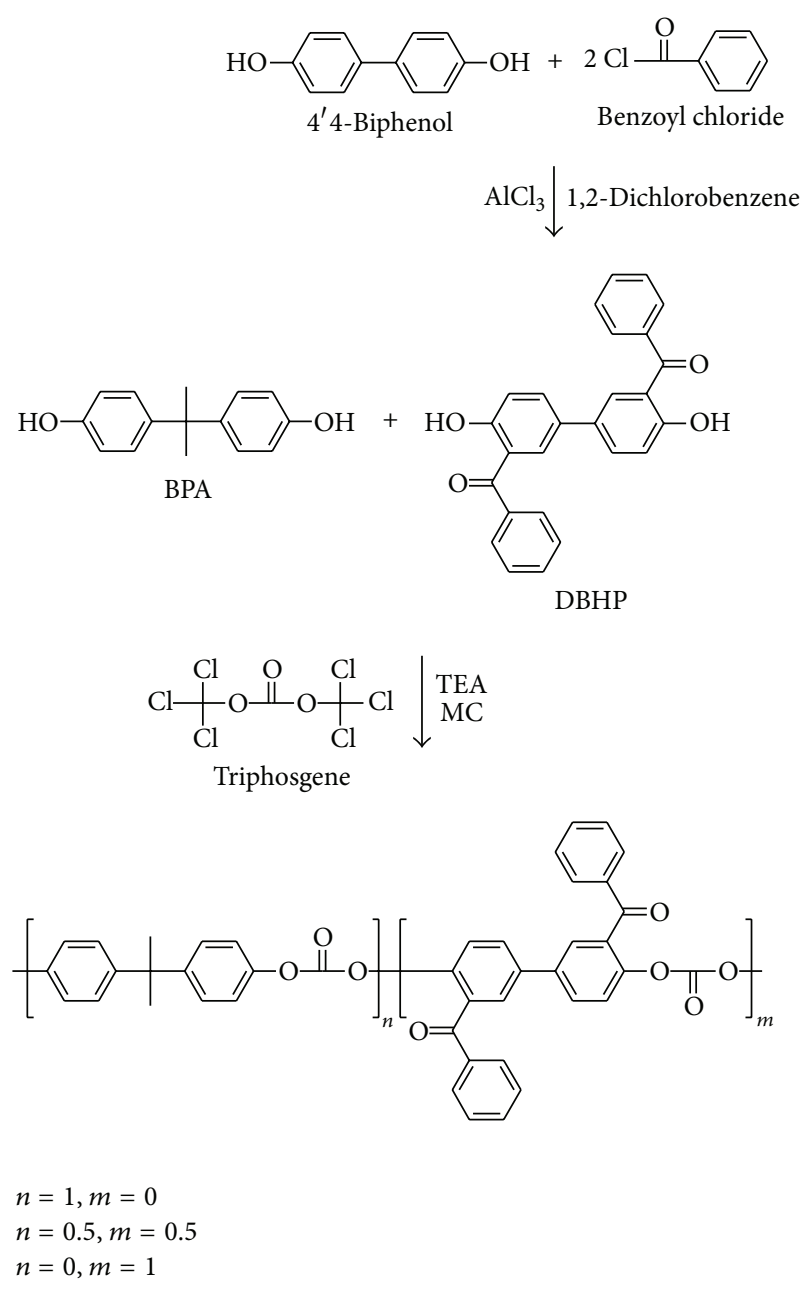

SCHEME 1: Synthesis of DBHP monomer and DBHP-PCs.

Interfacial polymerization is a commercial reaction method between sodium hydroxide solution and organic layer containing chloride. Low-temperature polymerization uses an insoluble monomer with hydroxide functional group in a hydroxide solution. Trimethylamine was used for dissolving monomer and as the catalyst in the reaction. Moreover, this reaction was controlled by temperature, because of rapid reactivity. The polycarbonate copolymers were prepared by low-temperature esterification reaction and synthesized from DBHP (25\%, 50\%, 75\%, and $100 \% \mathrm{~mol})$, bisphenol-A, and triphosgene, as shown in Scheme 1. In this case, DBHP monomer did not dissolve in hydroxide solution because of the steric hindrance from the side chain. Therefore, polymers were synthesized by low-temperature polymerization method.

The chemical structure of monomer and polymers was identified by ${ }^{1} \mathrm{H}$ NMR shown in Figure 1 . The ${ }^{1} \mathrm{H}$ NMR spectra of DBHP and BPA-DBHP polycarbonate also confirmed the structure, because all the peaks of the protons appeared at the expected positions. In the DBHP monomer, the ortho two proton peaks of main phenyl rings appeared at $7.02 \mathrm{ppm}$ and the 6 protons of phenyl rings near the ketone groups appeared

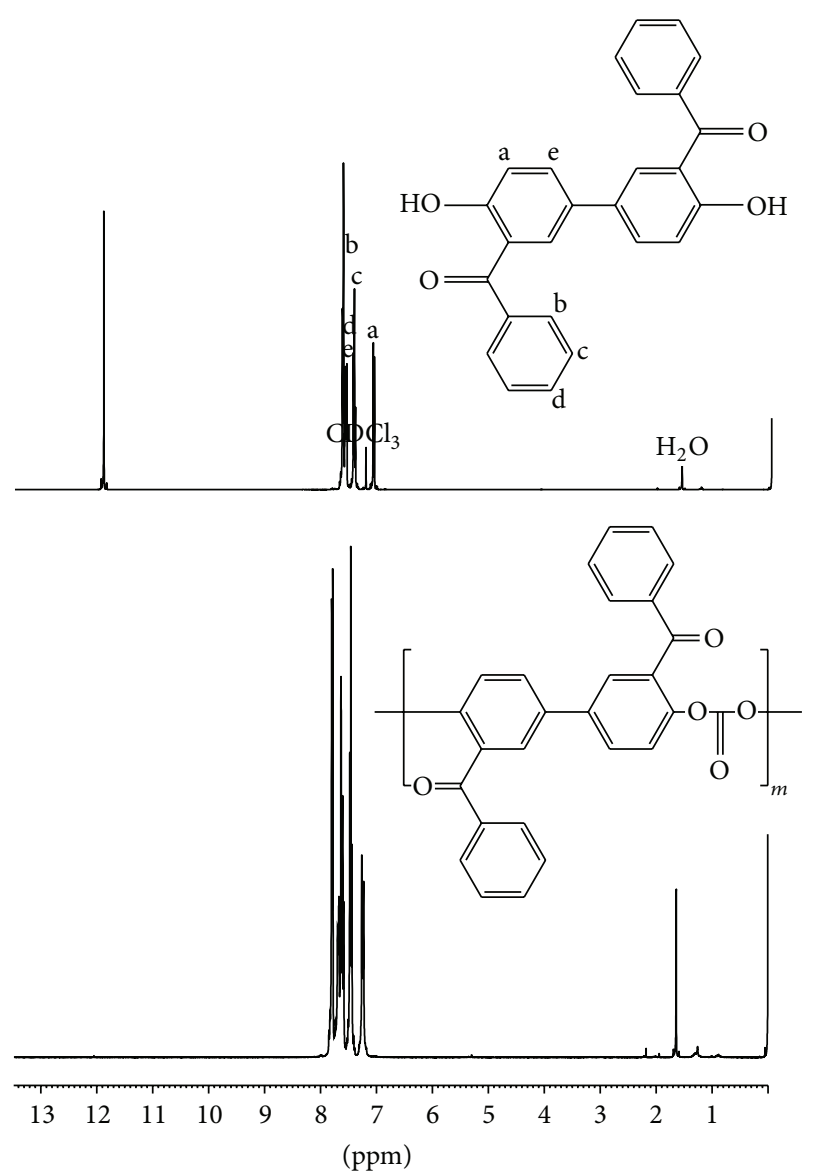

Figure 1: ${ }^{1} \mathrm{H}$ NMR of DBHP monomer and DBHP-PC.

at $7.6 \mathrm{ppm}$. The 2 proton peaks of the hydroxide group appeared at $12.4 \mathrm{ppm}$. The residual proton peaks appeared in the range $7.15-7.26 \mathrm{ppm}$. In the polymer, The 6 proton peaks of the BPA-methyl group appeared at $1.68 \mathrm{ppm}$, and the 8 proton peaks of BPA-phenyl rings appeared in the range 7.15$7.26 \mathrm{ppm}$. The ortho 2 proton peaks of the main phenyl rings appeared at $7.02 \mathrm{ppm}$, and the 6 protons of phenyl rings near the ketone groups appeared at $7.6 \mathrm{ppm}$. The residual proton peaks appeared in the range 7.15-7.26 ppm.

The thermal behavior of the polycarbonates was examined by differential scanning calorimetry (DSC). The glass transition temperature $\left(T_{q}\right)$ values of DBHP polycarbonates changed as increasing DBHP monomer segments. As shown in Figure 2, $T_{g}$ of DBHP's polymer appeared 143 and $148^{\circ} \mathrm{C}$, compared to $151^{\circ} \mathrm{C}$ for BPA-PC. $T_{g}$ values decreased with increasing content of DBHP, because the bulky benzoyl groups have more aromatic rings than BPA-PC, but the side phenyl rings have a large free volume and steric hindrance. The molecular weight of the polymers was determined by gel permeation chromatography (GPC). All the polymers showed a weight average molecular weight $\left(M_{w}\right)$ ranging from 54,400 to 68,100 , as listed in Table 1 .

The thermal stability of polymers was examined by thermogravimetric analysis (TGA). As shown in Figure 3, the initial weight loss of DBHP-PC copolymer started at 
TABLE 1: Properties of polycarbonates.

\begin{tabular}{lccccc}
\hline Polymers & $T_{g}\left({ }^{\circ} \mathrm{C}\right)$ & TGA $\left({ }^{\circ} \mathrm{C}, 5\right.$ wt $\%$ loss $)$ & $M_{w}$ & Contact angle $\left({ }^{\circ}\right)$ & Surface hardness \\
\hline BPA-PC & 151 & 495 & 54,400 & 96.3 & 63 \\
DBHP 25 & 148 & 431 & 58,200 & 103.2 & $5 \mathrm{~B}$ \\
DBHP 50 & 143 & 385 & 61,700 & 108.6 & $3 \mathrm{~B}$ \\
DBHP 75 & 145 & 372 & 64,900 & 112.3 & $2 \mathrm{~B}$ \\
DBHP 100 & 146 & 363 & 68,100 & 115.7 & $1 \mathrm{~B}$ \\
\hline
\end{tabular}

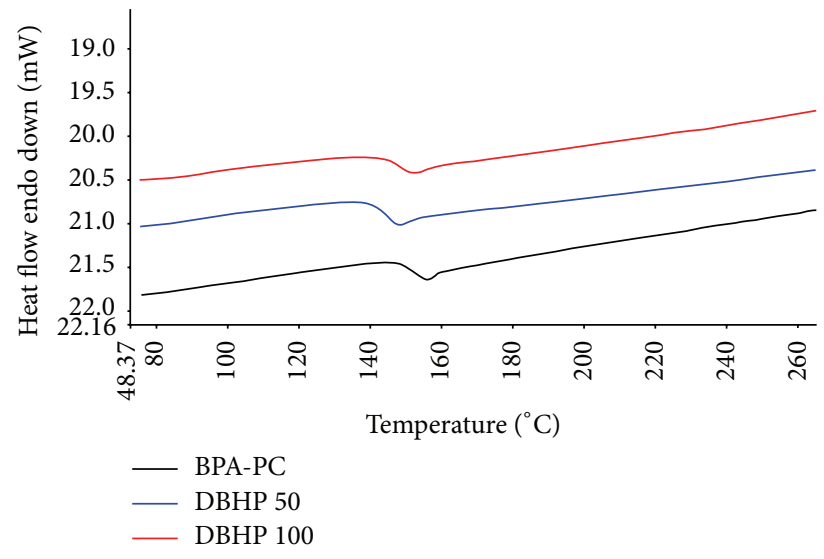

Figure 2: DSC of BPA-PC and DBHP-PCs.

$320 \sim 350^{\circ} \mathrm{C}$ and was assigned to the degradation start of the ketone of the side benzoyl group. The second decomposition at $580^{\circ} \mathrm{C}$ was attributed to the fragment of polymer main chain. The initial weight loss of DBHP polymer started in the range $320 \sim 400^{\circ} \mathrm{C}$ and was attributed to the degradation of the ketone of the side benzoyl group. The second decomposition at $430^{\circ} \mathrm{C}$ was attributed to the fragment of phenyl rings of the side chain benzoyl group. The third decomposition of $600^{\circ} \mathrm{C}$ was attributed to the splitting of the polymer main chain. DBHP-PC increased the thermal stability than BPAPC because of the presence of bulky side benzoyl groups and biphenyl rings.

The polymer's surface morphologies were assessed on $1 \mu \mathrm{m} \times 1 \mu \mathrm{m}$ scale by atomic force microscopy in the tapping mode under ambient conditions. Figure 4 showed microphase separation and was compared by the content of DBHP. Uneven surface was visible by the contained side phenyl groups. The bright and dark regions of the images correspond to the linear and dark areas, respectively, corresponding to the DBHP part. A common and useful method for determining the surface energy is to measure the contact angle of a water droplet on the surface.

The water droplet on a hydrophobic surface shows a higher contact angle, because of the lower surface energy against the surface tension of the droplet and vice versa. Figure 5 shows the images of the polymer of water drop on polymers' film. The synthesized DBHP had a higher contact angle than BPA-PC, and the curvature of the water surface was different, because of the content of DBHP, as listed in Table 1. BPA-PC had some hydrophilic part, because the carbonyl groups of BPA-PC have steric bulk, from the close to the

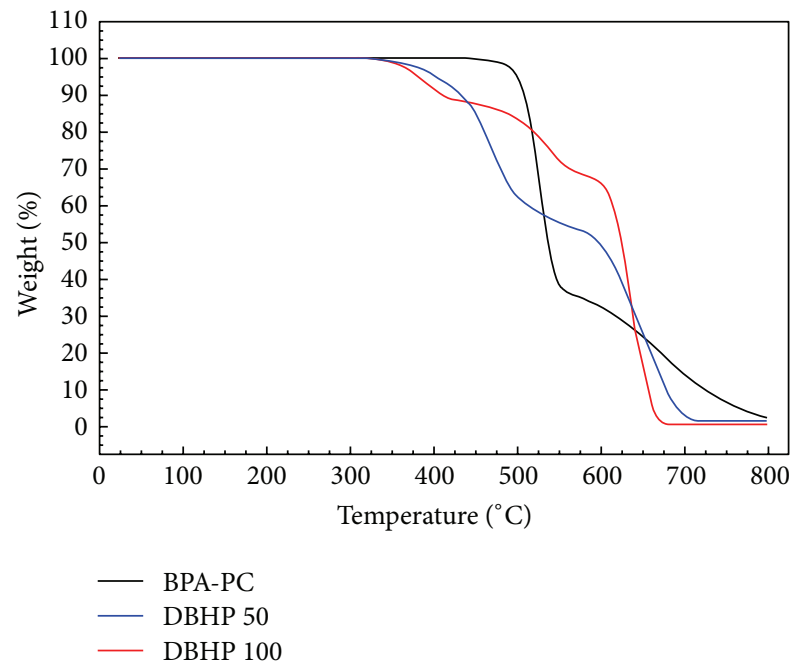

Figure 3: TGA of BPA-PC and DBHP-PCs.

surface. However, DBHP-PCs showed a lower surface resistance than BPA-PC, because DBHP have free-volume for the bulky structure of the side benzene. The surface hardness of polycarbonate sheet increased from B4 to B2; however, the hardness of a film with a thickness of $100 \mu \mathrm{m}$ was measured as $6 \mathrm{~B}$. The surface hardness of the synthesized copolymers was confirmed from $5 \mathrm{~B}$ to $1 \mathrm{~B}$ by content of DBHP, as listed Table 1 . According to research, polymers containing side ketone groups had a high mechanical performance with the surface hardness. The surface hardness of the polymers of DBHP increased, because of the side ketone groups.

The polycarbonates showed transparency as shown in Figure 6, compared to BPA-PC. In our daily lives, transparency is affected at $550 \mathrm{~nm}$ as an important part. DBHP 75 and 100 have a transparency of $75 \%$ and $79 \%$, lower than BPAPC of $85 \%$. Moreover, this polymer was confirmed as a rough surface like outer glass. Transparency of DBHP 25 and 50 was similar to those of BPA-PC and polymer film and was clear without a rough surface.

\section{Conclusions}

Polycarbonate copolymers were synthesized by low-temperature esterification reaction with DBHP, bisphenol-A, and triphosgene. The DBHP exhibited lower $T_{g}$ than linear polycarbonate. The polymer showed satisfactory thermooxidative stability. The surface morphologies of the films of the synthesized copolymer were also different. The surface hardness 

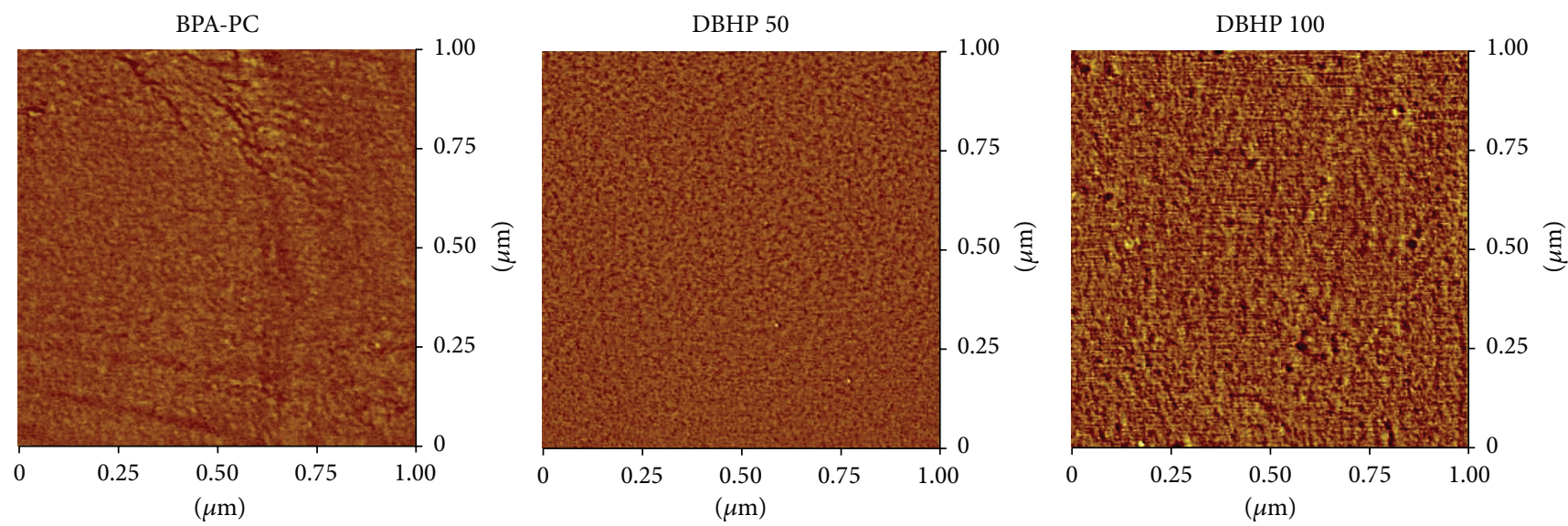

FIGURE 4: AFM of BPA-PC and DBHP-PCs.

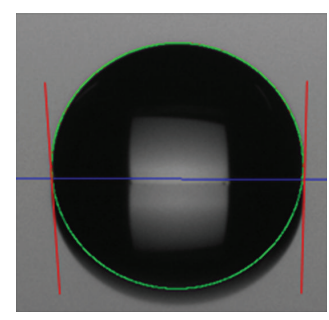

BPA-PC

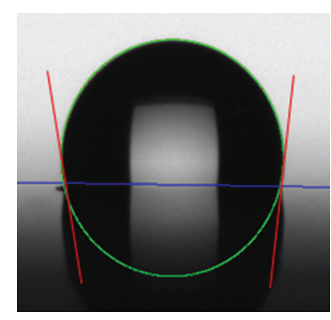

DBHP 25

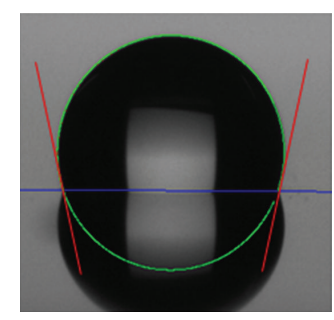

DBHP 50

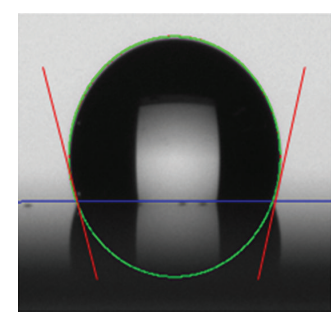

DBHP 75

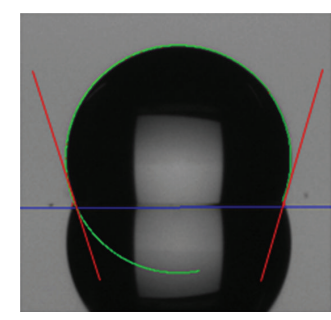

DBHP 100

FIGURE 5: Image of water drop on the polymers' film.

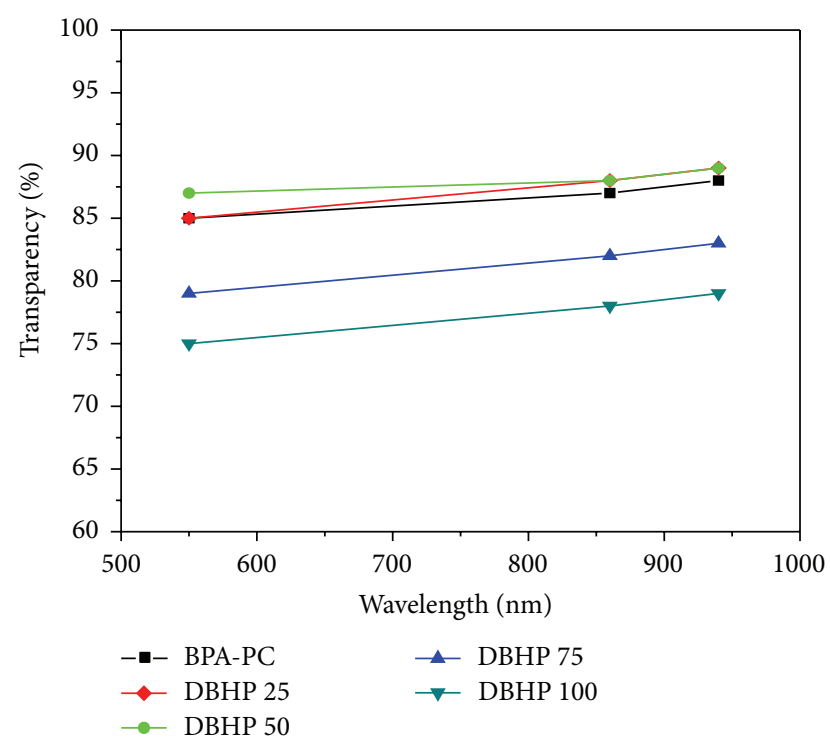

FIGURE 6: Transparency of polymers.

of the copolymers was measured from $5 \mathrm{~B}$ to $1 \mathrm{~B}$, compared to $6 \mathrm{~B}$ of $\mathrm{BPA}$ polycarbonate. Moreover, the transparency of polymers was measured from 75 to 89 , and DBHP 50 had a similar value as that of BPC-PC. This encouraging result was demonstrated to be used for engineering plastic's application.

\section{Competing Interests}

The authors declare that there is no conflict of interests regarding the publication of this paper.

\section{Acknowledgments}

This work was supported by the Human Resource Training Program for Regional Innovation and Creativity through the Ministry of Education and National Research Foundation of Korea (NRF-2014H1C1A1066447) and by the National Research Foundation of Korea (NRF) grant funded by the Korea government (MSIP) (2016R1A2B4010600).

\section{References}

[1] H. T. Pham, S. Munjal, and C. P. Bosnyak, "Polycarbonates," in Handbook of Thermoplastics, O. Olabisi, Ed., Marcel Dekker, New York, NY, USA, 1997.

[2] T. M. Madkour, "Polycarbonate," in Polymer Data Handbook, J. E. Mark, Ed., Oxford University Press, New York, NY, USA, 1999.

[3] H. T. Pham, C. L. Weckle, and J. M. Ceraso, "Rheology enhancement in PC/ABS blends," Advanced Materials, vol. 12, no. 23, pp. 1881-1885, 2000.

[4] H. Schnell, Chemistry and Physics of Polycarbonates, Wiley Interscience, New York, NY, USA, 1964. 
[5] C. Nguyen and J. Kim, "Synthesis of a novel nitrogen-phosphorus flame retardant based on phosphoramidate and its application to PC, PBT, EVA, and ABS," Macromolecular Research, vol. 16, no. 7, pp. 620-625, 2008.

[6] S. Krishnan and R. J. White, U.S. Patent 4,772,655, 1988.

[7] P. P. Policastro, P. K. Hernandez, G. C. Davis, and J. D. Rich, U.S. Patent 4,916,194, 1990.

[8] L. N. Lwis and S. C. Bunnel, U.S. Patent 4,954,549, 1990.

[9] H. L. Vincent, D. J. Kimball, and R. R. Boundy, "Polysiloxane/ silica hybrid resins abrasion resistant coatings for plastic substrates," Polymeric Materials: Science and Engineering, vol. 50, pp. 143-146, 1984.

[10] T. Ebeling, M. M. Marugan, Z. Qu, and S. Siripurapu, European Patent EP 1624025 A1, 2005.

[11] K. Horisawa, K. Okada, and W. Zhou, WO Patent 152741 Al, 2008.

[12] M. Okamoto, "Effect of polycarbonate-poly(methyl methacrylate) graft copolymer as a modifier improving the surface hardness of polycarbonate," Journal of Applied Polymer Science, vol. 83, no. 13, pp. 2774-2779, 2002.

[13] S. A. Xu and S. C. Tjong, "Tensile deformation mechanisms of the blends of polycarbonate with poly(methyl methacrylate)," European Polymer Journal, vol. 34, no. 8, pp. 1143-1149, 1998.

[14] L. R. Hutchings, R. W. Richards, R. L. Thompson, A. S. Clough, and S. Langridge, "Interface development in polycarbonate/ poly(methyl methacrylate) bilayer films," Journal of Polymer Science, Part B: Polymer Physics, vol. 39, no. 20, pp. 2351-2362, 2001.

[15] W.-P. Hsu, "Solvent effects on the miscibility of poly(methyl methacrylate)/poly(bisphenol A carbonate) blends," Journal of Applied Polymer Science, vol. 80, no. 14, pp. 2842-2850, 2001.

[16] K. K. Park, H. J. Lee, E. H. Kim, and S. K. Kang, "Facile synthesis and photo-Fries rearrangement of 2-benzoyl-4-benzoyloxyphenol leading to dibenzoyldihydroxybenzene derivatives," Journal of Photochemistry and Photobiology A: Chemistry, vol. 159, no. 1, pp. 17-21, 2003.

[17] A. M. A. G. Oliveira, A. M. F. Oliveira-Campos, M. M. M. Raposo, J. Griffiths, and A. E. H. Machado, "Fries rearrangement of dibenzofuran-2-yl ethanoate under photochemical and Lewis-acid-catalysed conditions," Tetrahedron, vol. 60, no. 29, pp. 6145-6154, 2004.

[18] M. R. Mauricio, T. D. S. Silva, M. H. Kunita, E. C. Muniz, G. M. De Carvalho, and A. F. Rubira, "Synthesis of luminescent polycarbonate grafted with methyl methacrylate/europium complex using supercritical $\mathrm{CO}_{2}$ technology as a green chemistry method," Journal of Materials Science, vol. 47, no. 12, pp. 49654971, 2012.

[19] J. Jayaramudu, G. Siva Mohan Reddy, K. Varaprasad, E. R. Sadiku, S. Sinha Ray, and A. Varada Rajulu, "Effect of alkali treatment on the morphology and tensile properties of Cordia Dichotoma fabric/polycarbonate composites," Advances in Polymer Technology, vol. 32, no. 3, Article ID 21349, 2013.

[20] S. E. Morgan, R. Misra, and P. Jones, "Nanomechanical and surface frictional characteristics of a copolymer based on benzoyl1,4-phenylene and 1,3-phenylene," Polymer, vol. 47, no. 8, pp. 2865-2873, 2006. 

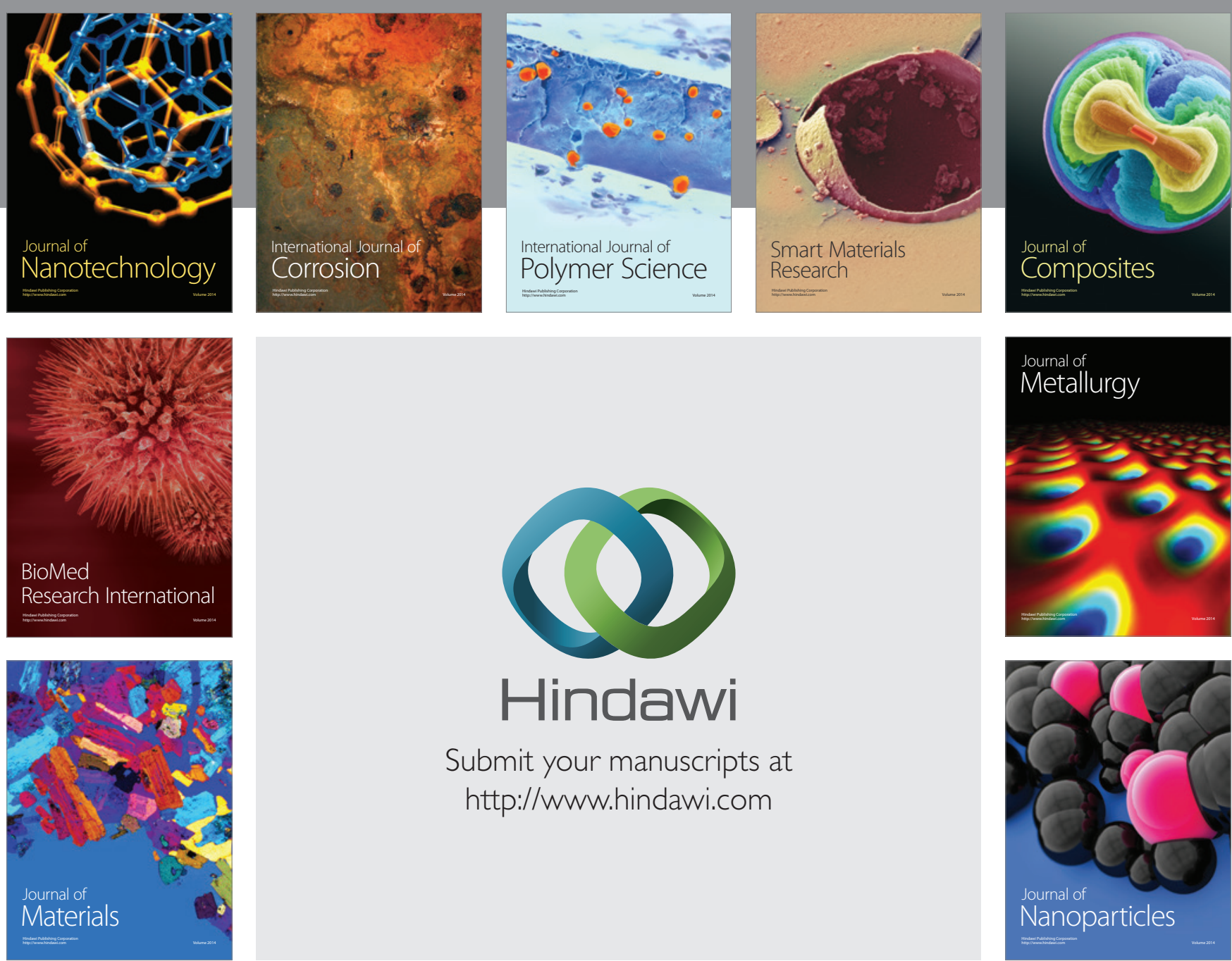

\section{Hindawi}

Submit your manuscripts at

http://www.hindawi.com

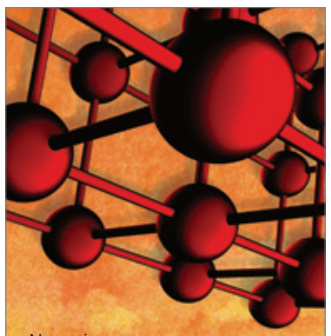

Materials Science and Engineering
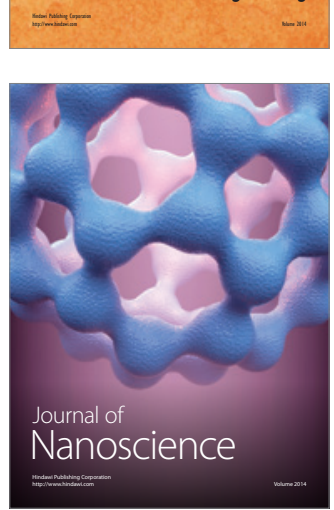
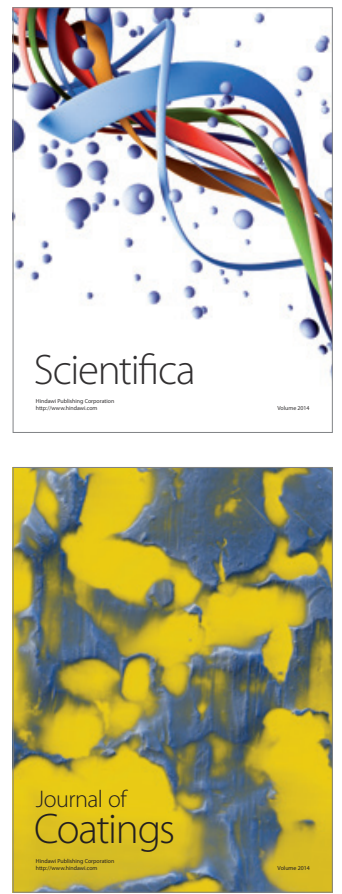
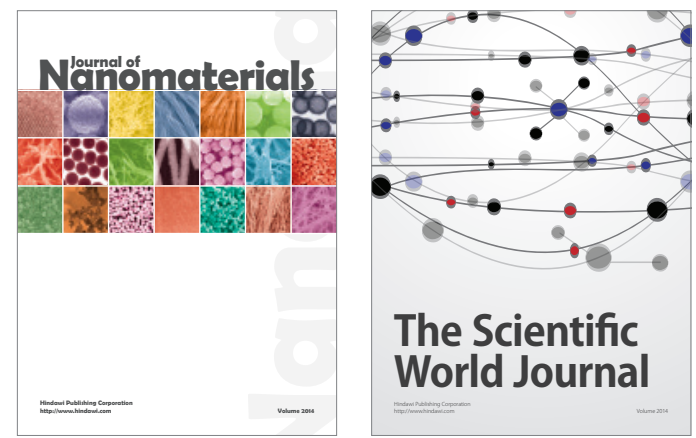

The Scientific World Journal
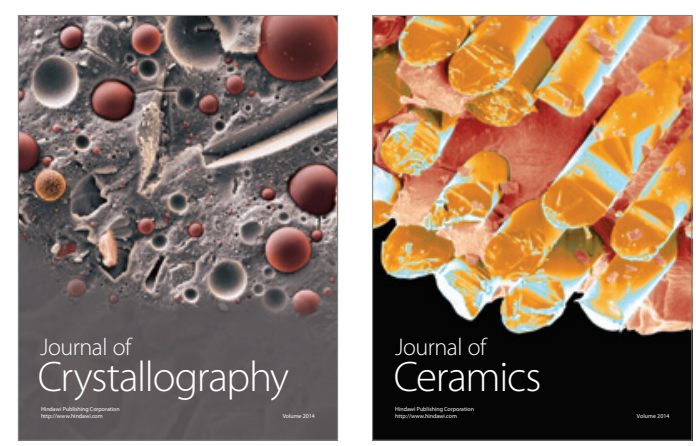
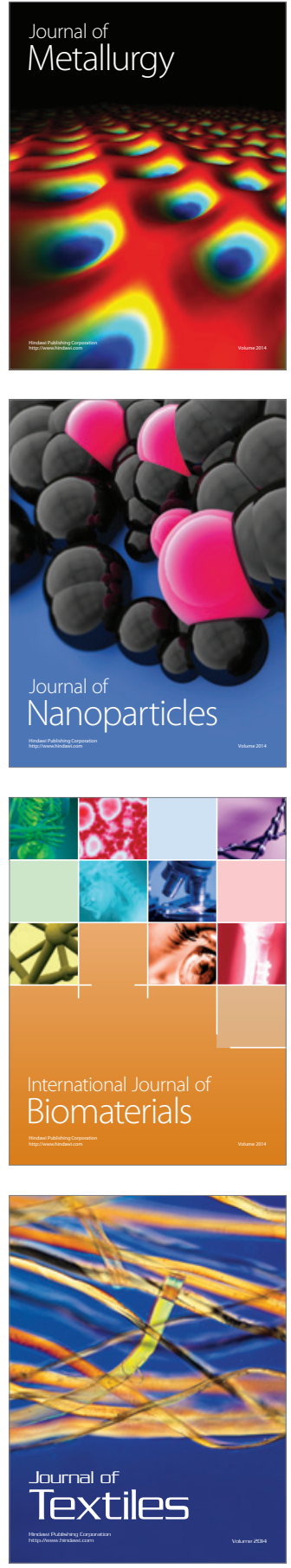